\title{
A simple ratio-based approach for power and sample size determination for 2-group comparison using Rasch models
}

\author{
Véronique Sébille ${ }^{1 *}$, Myriam Blanchin ${ }^{1}$, Francis Guillemin², Bruno Falissard ${ }^{3,4}$ and Jean-Benoit Hardouin ${ }^{1}$
}

\begin{abstract}
Background: Despite the widespread use of patient-reported Outcomes (PRO) in clinical studies, their design remains a challenge. Justification of study size is hardly provided, especially when a Rasch model is planned for analysing the data in a 2-group comparison study. The classical sample size formula (CLASSIC) for comparing normally distributed endpoints between two groups has shown to be inadequate in this setting (underestimated study sizes). A correction factor (RATIO) has been proposed to reach an adequate sample size from the CLASSIC when a Rasch model is intended to be used for analysis. The objective was to explore the impact of the parameters used for study design on the RATIO and to identify the most relevant to provide a simple method for sample size determination for Rasch modelling.

Methods: A large combination of parameters used for study design was simulated using a Monte Carlo method: variance of the latent trait, group effect, sample size per group, number of items and items difficulty parameters. A linear regression model explaining the RATIO and including all the former parameters as covariates was fitted.

Results: The most relevant parameters explaining the ratio's variations were the number of items and the variance of the latent trait $\left(R^{2}=99.4 \%\right)$.

Conclusions: Using the classical sample size formula adjusted with the proposed RATIO can provide a straightforward and reliable formula for sample size computation for 2-group comparison of PRO data using Rasch models.
\end{abstract}

Keywords: Patient-reported outcomes, Item response theory, Rasch model, Sample size, Power

\section{Background}

Patient-reported outcomes (PRO) are increasingly used in clinical research; they have become essential criteria that have gained major importance especially in chronically ill patients. Consequently, nowadays these outcomes are often considered as main secondary endpoints or even primary endpoints in clinical studies [1-4]. Two main types of analytic strategies are used for PRO data: so-called classical test theory (CTT) and models coming from Item Response Theory (IRT). CTT relies on the observed scores (possibly weighted sum of patients items' responses) that are assumed to provide a good representation of a "true" score, while IRT relies on an

\footnotetext{
*Correspondence: veronique.sebille@univ-nantes.fr

'EA 4275, Biostatistics, Pharmacoepidemiology and Subjective Measures in Health Sciences, University of Nantes, Nantes, France

Full list of author information is available at the end of the article
}

underlying response model relating the items responses to a latent trait, interpreted as the true individual quality of life (QoL) for instance. The potential of IRT models for constructing, validating, and reducing questionnaires and for analyzing PRO data has been regularly underlined [5-7]. IRT and in particular Rasch family models [8] can improve on the classical approach to PRO assessment with advantages that include interval measurements, appropriate management of missing data [9-11] and of possible floor and ceiling effects, comparison of patients across different instruments [12]. Consequently, many questionnaires are validated (or revalidated) using IRT along with CTT [13-15] allowing analysing PRO data with IRT models in clinical research.

Clinical research methodology has reached a high level of requirements through the publication of international guidelines including the CONSORT statement, the STROBE 
(Strengthening the Reporting of Observational Studies in epidemiology), or TREND (Transparent Reporting of Evaluations with Nonrandomized Designs), initiative for instance [16-19]. All of these published recommendations are aimed at improving the reporting of scientific investigations coming either from randomized clinical trials or observational studies and systematically include an item related to sample size justification and determination. Furthermore, good methodological standards recommend that methods used for sample size planning and for subsequent statistical analysis should be based on similar grounds. Even if guidelines have also been recently published for PRO based studies [20,21], the reporting of such studies often lacks mentioning the justification of study size and its computation. Three main types of situations are often encountered in 2group comparison studies: i) sample size determination is not performed whatever the intended analysis for PRO data (CTT and/or IRT), ii) tentative justification is occasionally given a posteriori for the size of studies, iii) sample size computation is made a priori but only relies on CTT (mostly using the classical formula for comparing normally distributed endpoints on expected mean scores) even if IRT models are envisaged for data analysis. In this latter case, previous studies have shown that the classical formula was inadequate for IRT models because it leads to underestimation of the required sample size [22]. From this perspective, a method has been recently developed for power and sample size determination when designing a study using a PRO as a primary endpoint when IRT models coming from the Rasch family are intended to be used for subsequent analysis of the data [23]. This method, named Raschpower, provides the power for a given sample size during the planning stage of a study in the framework of Rasch models. It depends on the following parameters (that are a priori assumed and fixed): the parameters related to the items of the questionnaire (items' number $J$ and difficulties parameters $\delta_{j}, j=1$, ...,J), the variance of the latent trait $\left(\sigma^{2}\right)$ and the mean difference between groups on the latent trait $(\gamma)$. Some of these parameters are easily known a priori when planning a study (e.g. number of items) others are sometimes more difficult to reach (e.g. items difficulties, $\sigma^{2}, \gamma$ ) and initial estimates based on the literature or pilot studies are required. Besides, whether all these parameters have the same importance regarding sample size determination for Rasch models is unknown. The aim of our paper is to explore the relative impact of these parameters on sample size computation and to identify the most relevant to be used during study design for reliable power determination for Rasch models. Our main objective is to provide a simple method for sample size determination when a Rasch model is planned for analysing PRO data in a 2-group comparison study.

\section{Methods}

\section{The Rasch model}

In the Rasch model [8], the responses to the items are modelled as a function of a latent variable representing the so-called ability of a patient measured by the questionnaire (e.g. QoL, anxiety, fatigue...). The latent variable is often considered as a random variable assumed to follow a normal distribution. In this model, each item is characterized by one parameter ( $\delta_{\mathrm{j}}$ for the jth item), named item difficulty because the higher its value, the lower the probability of a positive (favourable) response of the patient to this item regarding the latent trait being measured.

Let us consider that two groups of patients are compared and that a total of $\mathrm{N}$ patients have answered a questionnaire containing J binary items. Let $\mathrm{X}_{\mathrm{ij}}$ be a binary random variable representing the response of patient $i$ to item $j$ with realization $x_{i j}, \theta_{i}$ be the realization of the latent trait $\Theta$ for this patient, and $\gamma$ the group effect defined as the difference between the means of the latent trait in the two groups.

For each patient, the probability of responding to each item is:

$$
\begin{aligned}
P\left(X_{i j}=x_{i j} \mid \theta_{i}, \delta_{j}\right) & =\frac{\exp \left\{\left(\theta_{i}+g_{i} \gamma-\delta_{j}\right) x_{i j}\right\}}{1+\exp \left(\theta_{i}+g_{i} \gamma-\delta_{j}\right)}, \\
i & =1, \ldots, \mathrm{N} \text { and } \mathrm{j}=1, \ldots, \mathrm{J}
\end{aligned}
$$

where $\delta_{j}$ represents the difficulty parameter of item $j$ and $g_{i}=0,1$ for patients in the first or second group, respectively. The latent variable $\Theta$ is usually a random variable following a normal distribution with unknown parameters $\mu$ and $\sigma^{2}$. Marginal maximum likelihood estimation is often used for estimating the parameters of the model.

\section{Sample size determination in the framework of the Rasch model - The Raschpower method}

We assume that we want to design a clinical trial using a given dimension of a PRO (e.g. the Mental Health dimension of the SF-36) as a primary outcome in a twogroup cross-sectional study. Let $\gamma$ (assumed $>0$ ) be the difference between the mean values of the latent trait (e.g. mental health) in the two groups and $\sigma^{2}$ the common variance of the latent trait in both groups. We assume that the study involves the comparison of the two hypotheses $\mathrm{H}_{0}: \gamma=0$ against the two-sided alternative $\mathrm{H}_{1}: \gamma \neq 0$. If we plan to use a Rasch model that includes a group effect $\gamma$ (Eq 1) to test this null hypothesis on the data that will be gathered during the study with a given power $1-\beta_{R}$ and type I error $\alpha$, determination of the required sample size can be made using an adapted formula that has been implemented in the Raschpower method [23]. This method is based on the power of the 
Wald test of group effect $\gamma$ for a given sample size and it is briefly described. To perform a Wald test, an estimate $\Gamma$ of $\gamma$ is required as well as its standard error. Since we are designing a study, some assumptions are made regarding the expected values of these parameters. More specifically, $\Gamma$ is set at the assumed value for the group effect, $\gamma$, and its standard error is obtained as follows: an expected dataset of the patient's responses is created conditionally on the planning values that are assumed for the sample size in each group, the group effect $\gamma$, the items difficulties $\delta_{j}$, and the variance of the latent trait $\sigma^{2}$. The probabilities and the expected frequencies of all possible response patterns for each group are computed with the statistical model that will be used for analyzing the data that will be gathered during the study: a Rasch model. The variance of the group effect $\hat{\operatorname{Var}}(\hat{\gamma})$ is subsequently estimated using a Rasch model including a group effect with $\delta_{j}$ and $\sigma^{2}$ fixed to their planned expected values.

The power $1-\beta_{\mathrm{R}}$ is then computed with the following formula:

$$
1-\beta_{\mathrm{R}} \approx 1-\Phi\left(\mathrm{z}_{1-\alpha / 2}-\frac{\gamma}{\sqrt{\hat{\mathrm{V}} \operatorname{ar}(\hat{\gamma})}}\right)
$$

where $\Phi$ is the cumulative standard normal distribution function and $z_{1-\alpha / 2}$ the percentiles of the standard normal distribution. $1-\beta_{R}$ is the power of the Wald test of group effect when a Rasch model is used to detect $\gamma$ at level $\alpha$. In practice, $\gamma, \sigma^{2}$, and the items' difficulties are unknown population parameters and initial estimates based on the literature or pilot studies are required for calculations.

\section{Relationship between the Raschpower method and the classical formula for manifest normal variables}

Using the same notations as before $(\gamma$ is the group effect and $\sigma^{2}$ is the common variance of the latent trait for both groups), we can also compute the required sample size per group $\left(\mathrm{N}_{\mathrm{C} 0}\right.$ for the first group and $\mathrm{N}_{\mathrm{C} 1}$ for the second group) using the classical formula for comparing normally distributed endpoints with a given power $1-\beta$ and a type I error $\alpha$ to detect the group effect $\gamma$ as follows [24]:

$$
N_{C 0}=\frac{(\mathrm{k}+1) \times \sigma^{2} \times\left(\mathrm{z}_{1-\alpha / 2}-\mathrm{z}_{1-\beta}\right)^{2}}{\mathrm{k} \times \gamma^{2}}
$$

Where $\mathrm{N}_{\mathrm{C} 1}=\mathrm{k} \times \mathrm{N}_{\mathrm{C} 0}$ (when $\mathrm{k}=1$, the sample sizes are assumed equal in both groups).

The power $1-\beta$ for detecting a difference between groups equal to $\gamma$ with a total sample size of $\mathrm{N}_{C 0}+\mathrm{N}_{C 1}$ and a type I error set to $\alpha$ can also be computed as:

$$
1-\beta=\Phi\left(\sqrt{\frac{\mathrm{kN}_{\mathrm{C} 0} \times \gamma^{2}}{(\mathrm{k}+1) \times \sigma^{2}}}-\mathrm{z}_{1-\alpha / 2}\right)
$$

Let us assume without loss of generality that $\mathrm{k}=1$, that is we expect that the samples sizes are equal in each group $\left(\mathrm{N}_{\mathrm{C} 0}=\mathrm{N}_{\mathrm{C1}}=\mathrm{N}_{\mathrm{g}}\right)$. It has been evidenced [23] that the sample size per group computed using this classical formula $\left(N_{g}\right)$ allowed obtaining a power of 1- $\beta$ at level $\alpha$ for CTT-based analysis but did not provide the same power for Rasch-based analysis, but a lower power, computed with the Raschpower method, namely $1-\beta_{R} \leq 1-\beta$ (Figure 1, RP(1). Thus, using this classical formula, the sample size required when a Rasch model is used has to be increased to reach the desired power of 1- $\beta$ (i.e. $N_{g}$ has to be increased).

It has been observed in a previous study that this increase could be easily computed using the following relationships:

- since $1-\beta_{R} \leq 1-\beta$, the sample size that provides a power of $1-\beta_{R}$ using the classical formula (Eq 3 and Figure $1, C F(2)$, say $\mathrm{N}_{c}$, is lower than $\mathrm{N}_{\mathrm{g}}$ and the ratio $\mathrm{Ra}=\frac{N_{g}}{N_{c}}$ (Figure 1, (3)) is therefore higher than 1 - previous observations [23] have shown that this ratio Ra remained stable for different values of $N_{g}$ and 1- $\beta_{R}$, given $\gamma, \mathrm{J}$ and items difficulties - it has been noticed that multiplying $\mathrm{N}_{\mathrm{g}}$ by this ratio gave a sample size of $N_{R}=N_{g} \times R a$ (Figure 1, (4) that could provide the desired power $1-\beta$ for Rasch modelling (Figure 1, RP(5)

Hence this ratio Ra depends on the well-known classical formula and can be used to provide sample size calculations for Rasch modelling.

\section{Simulations}

A simulation study has been performed in order to get more insight into the relationships between the parameters that are required when planning a study for power determination for a given sample size $\left(\gamma, \sigma^{2}, \delta_{j}, J\right)$ and the ratio Ra. A large number of cases $\left(10^{6}\right)$ were simulated with each case corresponding to a single parameter combination $\left(\gamma, \sigma^{2}, \delta_{j}, J, N_{g}\right)$. The parameters values were randomly drawn from continuous or discrete uniform distributions, $\mathrm{U}[\min -\mathrm{max}]$, for: the variance of the latent trait $\sigma^{2}(\mathrm{U}[0.25-9])$, the group effect $\gamma(\mathrm{U}[0.2 \mathrm{x} \sigma-0.8 \mathrm{x} \sigma])$, the number of items J (U[3-20]), and the sample size per group $\mathrm{N}_{\mathrm{g}}$ assumed to be equal in both groups (U[50500]). The items difficulty parameters $\delta_{j}, j=1, \ldots$, , were drawn from a centred normal distribution with variance $\sigma^{2}$ and set to the percentiles of the distribution. The Raschpower method was applied on each parameter combination and provided the power $1-\beta_{R}$ for Rasch modelling as well as the ratio Ra. Multiple linear regression was performed to assess the contribution of $\mathrm{N}_{\mathrm{g}}, \gamma, \mathrm{J}$, and $\sigma^{2}$ and the difficulty parameters $\delta \mathrm{j}, \mathrm{j}=1, \ldots, \mathrm{J}$ to the variation of the ratio $\mathrm{Ra}$. The effects of the difficulty 


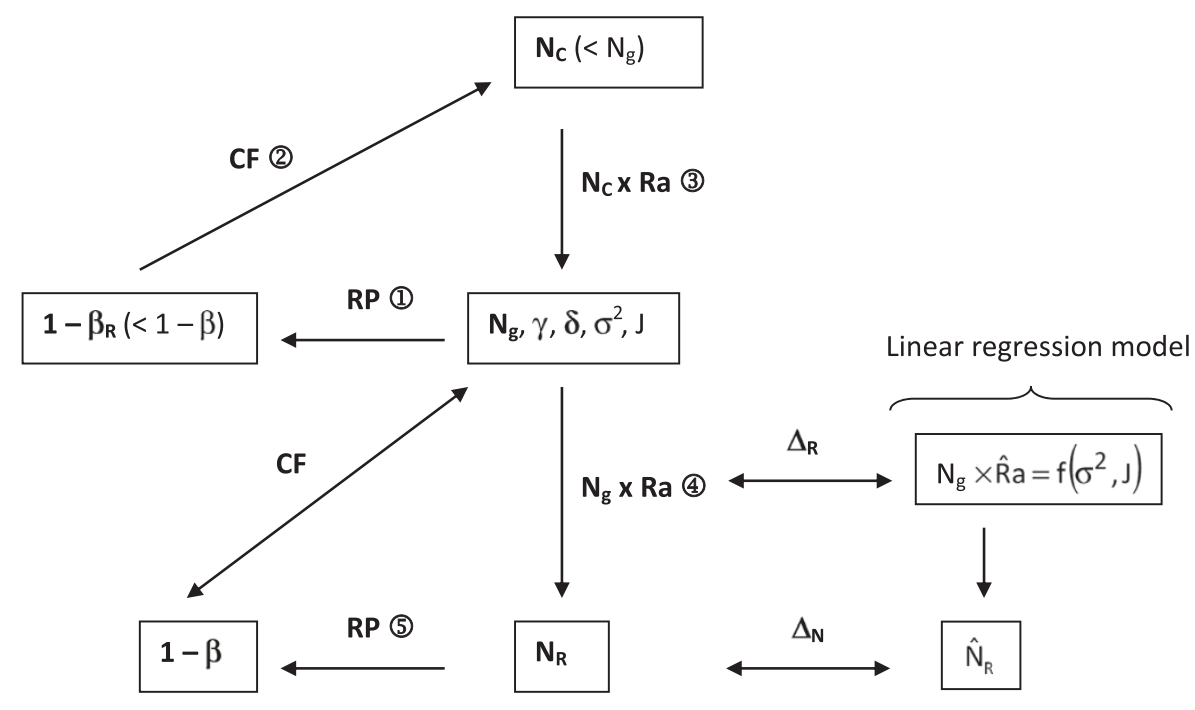

RP: Raschpower method; CF: classical formula for normally distributed variables

$\mathrm{N}_{\mathrm{g}}$ : sample size per group associated with a power of 1- $\beta_{\mathrm{R}}$ for Rasch analysis and 1- $\beta$ for CTTbased analysis; $\gamma$ : difference between the mean values of the latent trait in the two groups (group effect); $\delta$ : vector of the items parameters; $\sigma^{2}$ : variance of the latent trait; $N_{c}$ : sample size per group associated with a power of $1-\beta_{R}$ for CTT-based analysis; Ra: ratio computed with the Raschpower method; Ra: ratio predicted by the linear regression model; $\Delta_{\mathrm{R}}=\hat{\mathrm{Ra}}-\mathrm{Ra} ; \mathrm{N}_{\mathrm{R}}$ : sample size per group computed with the Raschpower method associated with a power of $1-\beta$ for Rasch analysis; $\hat{N}_{R}$ : sample size per group predicted by the linear regression model; $\Delta_{N}=\hat{N}_{R}-N_{R}$.

$\mathrm{J}$ : number of items; $\mathrm{N}_{\mathrm{R}}$ : sample size per group providing a $1-\beta$ power for Rasch analysis

Figure 1 Description of the whole procedure for power and sample size determination using the ratio with the Raschpower method and the linear regression model.

parameters on Ra were investigated in several ways for different values of $\mathrm{J}$ : i) by introducing each parameter individually $\delta_{j}, j=1, \ldots, J$, ii) by introducing their mean and variance. A two-tailed $\mathrm{P}$-value $<0.05$ was considered significant. The variance explained by the model $\left(R^{2}\right)$ and the root mean square error (RMSE) were obtained and contributed to variable selection. Variables were removed if $\mathrm{R}^{2}$ and RMSE remained stable (within a 0.01 range). Post-regression diagnoses were performed to ensure that all linear regression assumptions were met (normality and homoscedasticity of residuals). Statistical analysis was performed using SAS statistical software version 9.3 (SAS Institute Inc, Cary, North Carolina).

\section{Results}

Among the $10^{6}$ parameter combinations, 15278 corresponded to the largest power for CTT and Rasch-based analysis, $100 \%$, where the ratio cannot be computed.
Hence all analyses were performed on 984722 parameter combinations.

A full linear model explaining the value of Ra was first fitted including $\mathrm{N}_{\mathrm{g}}, \gamma, 1 / \mathrm{J}, 1 / \sigma^{2}$, the difficulty parameters

Table 1 Parameters estimates of the linear regression model explaining the ratio provided by the Raschpower method

\begin{tabular}{lcc}
\hline Variables & $\mathbf{N}_{\text {POP }}=\mathbf{9 8 4 7 2 2}$ & P-values \\
\hline Intercept & $1.012\left(7.010^{-5}\right)$ & $<10^{-3}$ \\
$1 / \sigma^{2}$ & $0.095\left(1.010^{-4}\right)$ & $<10^{-3}$ \\
$1 / \mathrm{J}$ & $0.939\left(5.010^{-4}\right)$ & $<10^{-3}$ \\
Interaction $\left(1 / \sigma^{2 *} 1 / \mathrm{J}\right)$ & $3.730\left(7.510^{-4}\right)$ & $<10^{-3}$ \\
$\mathrm{R}^{2}$ & 0.994 & $/$ \\
RMSE & 0.030 & $/$ \\
\hline
\end{tabular}

Standard errors in parentheses. $\sigma^{2}$ : variance of the latent trait; J: number of items. 
(included either individually or using their mean and variance) and their interactions. A backward procedure was used for variable selection relying on the $R^{2}$ and RMSE variations between models and not on p-values. Indeed, since the number of simulated combinations was high (984722), all parameters were significant but not necessarily meaningful (very small estimated values). The $\mathrm{R}^{2}$ and RMSE remained stable during the backward procedure until the final model only containing $1 / \mathrm{J}$ and $1 / \sigma^{2}$ and their interaction was obtained (a maximum variation of 0.0015 and of 0.0037 was observed for the $\mathrm{R}^{2}$ and the RMSE, respectively). The model that was retained can be written as follows:

$$
\begin{aligned}
\mathrm{Ra}_{\mathrm{i}}= & \beta_{0}+\left(\beta_{1} \times \frac{1}{\sigma_{\mathrm{i}}^{2}}\right)+\left(\beta_{2} \times \frac{1}{\mathrm{~J}_{\mathrm{i}}}\right) \\
& +\left(\beta_{3} \times \frac{1}{\sigma_{\mathrm{i}}^{2}} \times \frac{1}{\mathrm{~J}_{\mathrm{i}}}\right)+\varepsilon_{\mathrm{i}}
\end{aligned}
$$

where $\varepsilon_{\mathrm{i}} \sim \mathrm{N}\left(0, \sigma_{\varepsilon}^{2}\right)$, for $\mathrm{i}=1, \ldots, 984722$

Table 1 shows the estimates of the multiple linear regression model that explains $\mathrm{R}^{2}=99.4 \%$ of the variance of the ratio and displays high accuracy (RMSE $=0.030$ ). The interaction between $1 / \sigma^{2}$ and $1 / \mathrm{J}$ is significant; the effect of $1 / \sigma^{2}$ on the ratio seems to be more pronounced when $1 / J$ is large (i.e.: $J$ is small). The ratio increases with $1 / \sigma^{2}$ (ie: when $\sigma^{2}$ decreases) and with $1 /$ J (i.e.: when $\mathrm{J}$ gets smaller).

The number of subjects per group predicted by this model was computed as follows: $\hat{\mathrm{N}}_{\mathrm{R}}=\mathrm{N}_{\mathrm{g}} \times \hat{\mathrm{R} a}$ where $\hat{R} a$ is the ratio predicted by the model. It was compared
Table 2 Distributions of the difference between the ratio (respectively number of subjects per group) predicted by the model and the one expected by the Raschpower method $\Delta_{R}$ (respectively $\Delta_{N}$ ) and according to the

\begin{tabular}{|c|c|}
\hline Variables & $N_{\text {POP }}=1996077$ \\
\hline & $2.5 \%$ / Median / 97.5\% \\
\hline & [min-max] \\
\hline \multirow[t]{2}{*}{$\Delta_{R}$} & $-0.049 / 0.002 / 0.043$ \\
\hline & {$[-1.236 ; 0.230]$} \\
\hline \multirow[t]{3}{*}{$\Delta_{\mathrm{N}}$} & $-10.623 / 0.438 / 13.499$ \\
\hline & {$[-179.576 ; 112.064]$} \\
\hline & n (\%) \\
\hline - Thres $<\Delta_{N}<+$ Thres & $968364(98.34 \%)$ \\
\hline$\Delta_{N}<-$ Thres & $10865(1.10 \%)^{\S}$ \\
\hline$\Delta_{N}>+$ Thres & $5493(0.56 \%)^{\dagger}$ \\
\hline \multicolumn{2}{|c|}{$\begin{array}{l}\text { Thres : threshold corresponding to } 5 \% \text { of the number of subjects per group } \\
\text { derived from the Raschpower method. } \\
\varsigma \text { : underestimation of the number of subjects per group produced by the } \\
\text { model as compared to the Raschpower method; }{ }^{+} \text {: overestimation of the } \\
\text { number of subjects per group produced by the model as compared to the } \\
\text { Raschpower method. }\end{array}$} \\
\hline
\end{tabular}
threshold (Thres) for $\Delta_{\mathrm{N}}$

to the expected number of subject per group $\mathrm{N}_{\mathrm{R}}=\mathrm{N}_{\mathrm{g}} \times$ $\mathrm{Ra}$ where $\mathrm{Ra}$ is the ratio derived from the Raschpower method. The difference between the ratio (respectively number of subjects per group) predicted by the model $\hat{R} a$ (respectively $\hat{\mathrm{N}}_{\mathrm{R}}$ ) and the one associated with the Raschpower method Ra (respectively $N_{R}$ ) was computed

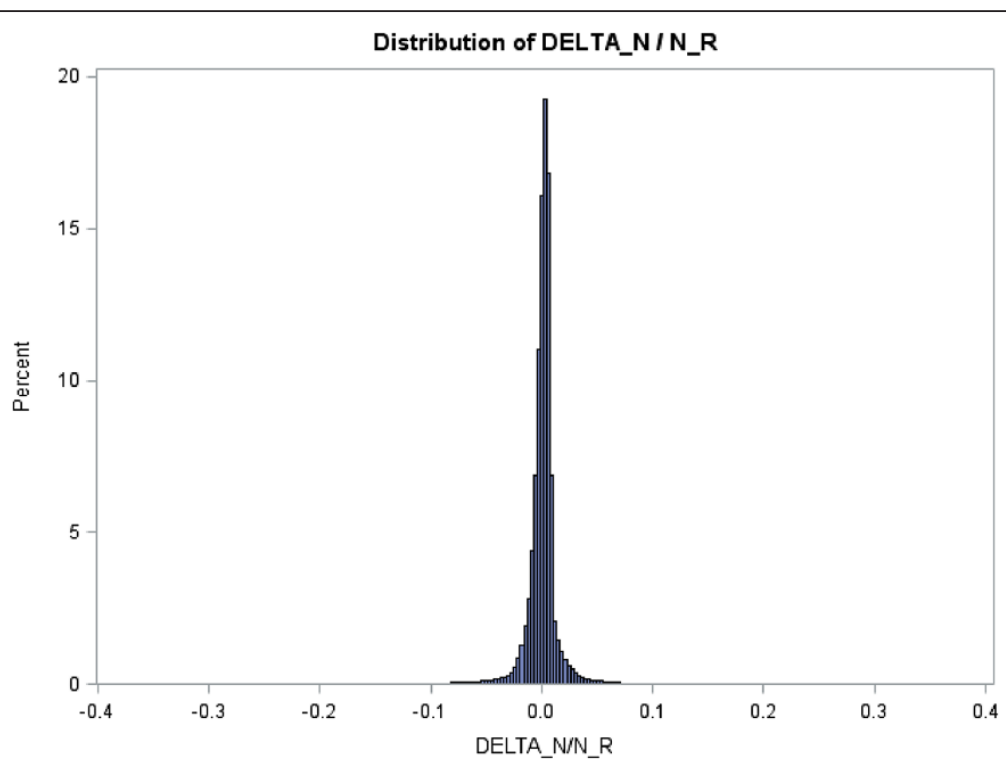

Figure 2 Distributions of $\Delta_{N} / N_{R}$ with $\Delta_{N}=\hat{N}_{R}-N_{R}$, where $\hat{N}_{R}$ is the number of subjects per group predicted by the linear regression model and $N_{R}$ is the number of subjects per group associated with the Raschpower method. 
for all parameters combinations with $\Delta_{\mathrm{R}}=\hat{\mathrm{R}} \mathrm{a}-\mathrm{Ra}$ and $\Delta_{\mathrm{N}}=\hat{\mathrm{N}}_{\mathrm{R}}-\mathrm{N}_{\mathrm{R}}$. Figure 2 shows the distributions of $\Delta_{\mathrm{N}} /$ $\mathrm{N}_{\mathrm{R}}$ which is distributed around 0 .

To quantify more precisely the magnitude of the difference $\Delta_{N}$, a threshold (Thres) corresponding to $5 \%$ of the number of subjects per group expected with the Raschpower method, Thres $=0.05 \times \mathrm{N}_{\mathrm{R}}$, was calculated for all parameters combinations. The descriptive statistics related to the distributions of $\Delta_{R}, \Delta_{N}$, and to $\Delta_{N}$ with respect to Thres are displayed in Table 2 . Ninetyfive percent of the values of $\Delta_{R}$, (respectively $\Delta_{\mathrm{N}}$ ) lie between -0.049 and 0.043 (respectively -10.623 and 13.499). The largest overestimation (respectively underestimation) of the number of subjects per group predicted by the model is about 112 subjects per group (respectively -180 subjects per group). The distribution of $\Delta_{\mathrm{N}}$ mostly lies (98.34\% of the cases) within the interval [-Thres - + Thres] corresponding to $\pm 5 \%$ of the number of subjects per group expected with the Raschpower method. Moreover, the model rarely predicted $(0.56 \%)$ an overestimated number of subjects per group of more than $5 \%$ of the sample size per group expected with Raschpower. This case only occurs when the variance of the latent trait $\sigma^{2}<1$ and $\mathrm{J}>7$ items. An underestimated number of subjects per group of more than $5 \%$ of the sample size per group expected with Raschpower is occasionally observed $(1.10 \%)$ and it mostly occurs (more than $90 \%$ of the cases) when $J$ is larger than 16 items and mostly when $\sigma^{2}<1$ ( $75 \%$ of the cases).

The whole procedure including the Raschpower method and the linear regression model for power and sample size determination using the ratio is summarized in Figure 1.

\section{An example of sample size determination in clinical research using the ratio - NHP data}

The data come from a pilot study whose main objective is to compare the pain level of two groups of patients having either Steinert's disease or another muscular dystrophy. The two disease groups have similar symptoms but also present a number of dissimilar features such as pain, cognitive disorders or male hypogonadism that are more frequently encountered in patients suffering from Steinert's disease and may impact QoL. Since QoL and in particular pain assessment may help to better understand the burden of disease from the patients' perspective and improving health outcomes and management, the pain dimension of the Nottingham Health Profile (NHP) questionnaire was used; it is composed of eight binary items $(J=8)$. The ethics committee of Reims, France granted approval for the study and patients were recruited in the university hospital of Reims: 52 patients were included with Steinert's disease and 95 patients with others muscular dystrophies. A Rasch model including a group effect $\gamma$ was fitted on these data and its global fit was not rejected by the $R_{1 \mathrm{~m}}$ test $(\mathrm{p}=0.329)$ [25]. The estimation of the difference between the means of the latent trait of the two groups was $\hat{\gamma}=0.649$ and the estimated latent trait's variance was $\hat{\sigma}^{2}=3.9323$ (non-significant difference between groups: $\mathrm{p}=0.08$ ). The objective was to use this pilot study to help planning a future possibly larger study that would provide enough power to detect this difference on the latent trait using a Rasch model. Indeed, it seemed valuable to the clinicians to determine a sample size large enough to be able to significantly detect this difference considered as clinically relevant with a power of $1-\beta=90 \%$ using a Rasch model. The sample size per group computed using the classical formula (Eq 3), for detecting $\gamma=$ 0.649 with a $90 \%$ power at $\alpha=5 \%$, assuming $\sigma^{2}=3.9323$, is $\mathrm{N}_{\mathrm{g}}=197$ for CTT-based analysis. We know that $\mathrm{N}_{\mathrm{g}}$ has to be increased to reach the desired power for Rasch modelling using the ratio. The ratio predicted by the multiple linear regression model can be easily computed as follows using the values of $\mathrm{J}$ and $\sigma^{2}$ :

$$
\begin{aligned}
\hat{\mathrm{R}} \mathrm{a}= & 1.012+\left(0.095 \times \frac{1}{3.9323}\right) \\
& +\left(0.939 \times \frac{1}{8}\right)+\left(3.730 \times \frac{1}{3.9323} \times \frac{1}{8}\right) \\
= & 1.27210
\end{aligned}
$$

Multiplying $\mathrm{N}_{\mathrm{g}}$ by this ratio gives a sample size of $\hat{\mathrm{N}}_{\mathrm{R}}=$ $197 \times 1.27210 \approx 251$ patients per group that should provide the desired power of $90 \%$ for Rasch modelling of the pain dimension of the NHP questionnaire. These results were compared to those obtained with the Raschpower

Table 3 Comparison of the required parameters and the

\begin{tabular}{|c|c|c|}
\hline Variables & Linear regression model & Raschpower method \\
\hline$\overline{\sigma^{2}}$ & 3.9323 & 3.9323 \\
\hline$J$ & 8 & 8 \\
\hline Y & / & 0.649 \\
\hline $\mathrm{N}_{\mathrm{g}}$ & / & 197 \\
\hline$\delta$ & / & $\begin{array}{c}(2.61,2.94,1.75,0.46 \\
-0.11,0.36,1.28,2.23)\end{array}$ \\
\hline $\mathrm{Ra}$ & 1.27210 & 1.34 \\
\hline$N_{R}$ & 251 & 264 \\
\hline
\end{tabular}
results obtained using the linear regression model and the Raschpower method on the NHP data

$\sigma^{2}$ : variance of the latent trait; J: number of items; $ү$ : difference between the mean values of the latent trait in the two groups (group effect); $\mathrm{N}_{\mathrm{g}}$ : sample size per group providing a $1-\beta=90 \%$ power with the classical formula and a $1-\beta_{R}=80 \%$ power with Raschpower; $\delta$ : vector of the items parameters $\left(\delta_{1}, \delta_{2}\right.$, $\left.\delta_{3}, \delta_{4}, \delta_{5}, \delta_{6}, \delta_{7}, \delta_{8}\right) ;$ Ra: ratio, $N_{R}$ : sample size per group providing a $1-\beta \approx 90 \%$ power for Rasch analysis with the linear regression model and Raschpower, /: not required. 
method using the estimated difficulty parameters from the pilot study $(2.61,2.94,1.75,0.46,-0.11,0.36,1.28,2.23)$, $\hat{\gamma}=0.649, \hat{\sigma}^{2}=3.9323$, and $\mathrm{N}_{\mathrm{g}}=197$ per group. An $80 \%$ power $\left(1-\beta_{\mathrm{R}}\right)$ is expected using the Raschpower method for Rasch modelling with a sample size of $\mathrm{N}_{\mathrm{g}}=197$ per group (Figure 1, RP(1). The proposed ratio is therefore equal to $197 / 147=1.34$ where $\mathrm{N}_{\mathrm{c}}=147$ is the number of subjects per group that provides a power of $80 \%$ using the classical formula (Figure 1, CF(2). Hence, using the ratio, $197 \times 1.34 \approx 264\left(\mathrm{~N}_{\mathrm{R}}\right)$ patients per group should provide the desired power of $90 \%$ for Rasch modelling (Figure 1, RP(5)).

The parameters that are required for the determination of the ratio using the linear regression model or the Raschpower method as well as their corresponding values appear in Table 3. The ratio provided by the linear regression model and Raschpower (Table 3) are close to one another $\left(\Delta_{R}=-0.0679\right)$ and the number of subjects per group are $\left|\Delta_{\mathrm{N}}\right|=13$ patients apart. Moreover, since $\left|\Delta_{N}\right| / N_{R}=13 / 264=0.0492$, the linear model's prediction was within $5 \%$ of the expected sample size provided by the Raschpower method.

\section{Discussion}

Our results revealed that the sample size required in the framework of two-group cross-sectional studies for subsequent use of a Rasch model to analyse PRO data can be easily computed using the classical formula for comparing normally distributed endpoints along with a correction factor (named ratio in this paper). The most relevant parameters explaining this ratio's variation $\left(R^{2}=99.4 \%\right)$ were the number of items of the questionnaire to be used in the study $(J)$ and the latent trait's variance $\left(\sigma^{2}\right)$. Hence when designing a study, the most important parameters for reliable power determination using this ratio when a Rasch model is intended to be used to analyse PRO data appear to be the variance of the latent trait and the number of items regardless of the values of the group effect $(\gamma)$ and items parameters $\left(\delta_{j}, j=1, \ldots, J\right)$. A preliminary investigation had already evidenced that the precision with which item difficulty parameters were known did not have an impact on power determination of the test of group effect using a Rasch model [22]. However, in this previous study, the number of items J greatly impacted power as it was observed in our current study for sample size determination; both (sample size and power) being very closely related. The power increased with $J$ in line with what we observed in this study where the ratio decreased when J rose from 3 to 20 items, implying that fewer subjects were needed to obtain the same power when $J=20$ as compared to $J=3$. Moreover, this decrease of the ratio was more marked as $\sigma^{2}$ got smaller (significant interaction between $1 / \mathrm{J}$ and $\left.1 / \sigma^{2}\right)$. Quite a large range of values were

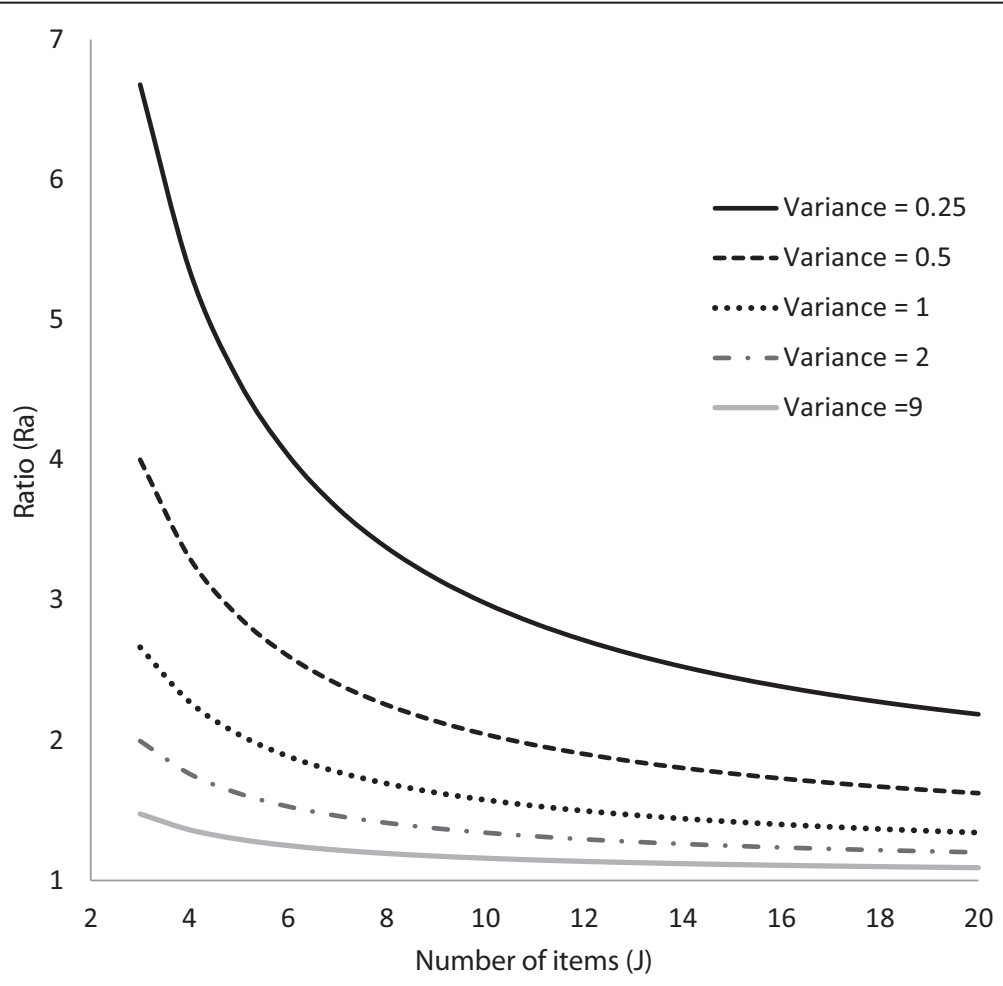

Figure 3 Values of the ratio $\mathrm{Ra}$ as a function of the number of items $\mathrm{J}$ according to the values of the variance of the latent trait. 
chosen for the variance of the latent trait (from 0.25 to 9) and for the number of items J (from 3 to 20) that allowed investigating more in depth the magnitude of their impact on the ratio. Figure 3 shows the evolution of the ratio $\mathrm{Ra}$ as a function of the number of items $J$ according to the values of the variance of the latent trait $\sigma^{2}$. The effect of $\sigma^{2}$ on the ratio was large, especially for small values of the variance $\left(\sigma^{2}<1\right)$, the ratio increasing as $\sigma^{2}$ decreased. This result, which might be thought as counter-intuitive, comes from the fact that the ratio, used to correct the sample size coming from the classical formula to obtain an adequately powered Rasch model, is a measure of the distance between the sample sizes corresponding to the powers expected for CTT and Rasch-based analyses. This distance becomes larger as the variance gets smaller and it reaches its maximum when $\sigma^{2}<1$. Hence, the correction factor (ratio) is likely to get larger as $\sigma^{2}$ decreases and the distance between the sample sizes for CTT and Rasch increases. Furthermore, it can be noted that when $\sigma^{2}<1$, the linear regression model could predict an overestimated number of subjects per group of more than $5 \%$ of the sample size per group expected with Raschpower (in at most $0.56 \%$ of all parameters combinations). An underestimation of more than $5 \%$ of the sample size per group expected with Raschpower could also be noticed (in at most $1.10 \%$ of all parameters combinations) for small values of $\sigma^{2}\left(\sigma^{2}<1\right.$ in $75 \%$ of the cases) and large values of $J(J>17$ in more than $90 \%$ of the cases). It can be emphasized that such small variances for the latent trait might be rarely encountered in practice especially when J is large $[26,27]$; hence this simple regression model should be reliable for sample size determination in most situations usually found in clinical research. Nevertheless, one of the major issues regarding study design and sample size determination still remains: to what expected values should we fix the key parameters? In our case, the challenge is put on one single parameter, the expected value for the variance of the latent trait. Retrospective, pilot data or published studies can be used for that purpose to provide information regarding the plausible range of values for the variance. However, it can turn out to be problematic if no previous studies can provide this information and it seems important to further study the impact of misspecifications of the planning values for the variance on the performance of the proposed method for sample size determination for Rasch modelling.

The fact that the number of subjects given by the classical formula, based on the latent trait, has to be increased using the ratio to reach the expected power for Rasch modelling could deliver a wrong message. Indeed, it could be interpreted as if Rasch models required more subjects than CTT-based analyses would. In fact, the classical formula is directly computed from the expected difference between the latent traits in both groups and the latent trait's variance in each group, assumed to be equal. By doing so, we assume that the means and variance of the latent traits are "perfectly" known and thus do not take into account the fact that the latent trait is not an observed (manifest) variable. Hence, its estimation requires the use of a model which creates uncertainty, unlike scores that can be directly observed and measured. This uncertainty is taken into account by adjusting the sample size using the ratio to obtain an adequately sized study for Rasch modelling. Moreover, it has been underlined that the so-called effect size (difference in means over the standard deviation) on the score scale was lower than the corresponding effect size on the latent trait scale. Consequently, the sample size requested for CTT-based analysis using the effect size on the score scale is higher than its counterpart on the latent trait scale.

The proposed method can be used with confidence when J stands between 3 and 20 and especially when the variance of the latent trait is expected to be higher than 1 . Otherwise (when $\sigma^{2}<1$ ), the Raschpower method should be preferred since the ratio-based approach might under or overestimate the sample size. One of the limitations of our study is that we focused on one of the most wellknown IRT model, the Rasch model. The Raschpower method has also been developed for other models that are well suited for the analysis of polytomous item responses, such as the Partial Credit Model or the Rating Scale Model (Hardouin, under revision). Moreover, the Raschpower method has recently been extended to deal with longitudinal designs [28] and it might be expected that this ratio would also be worthwhile in these contexts. Finally, the Raschpower method (for dichotomous and polytomous items and for cross-sectional and longitudinal designs) and the ratio-based approach (for dichotomous items) have been implemented in the free Raschpower module available at the website PRO-online http://proonline.univ-nantes.fr.

\section{Conclusion}

Using the classical formula for normally distributed endpoints along with the proposed ratio only depending on the number of items and the variance of the latent trait can provide a straightforward and reliable formula for sample size computation for subsequent Rasch-based analysis of PRO data.

\section{Competing interests}

The authors declare that they have no competing interests.

\section{Authors' contributions}

VS, JBH and MB have made substantial contributions to conception and design, analysis and interpretation of data; BF and FG has been involved in drafting the manuscript and revising it critically for important intellectual content. All authors read and approved the final manuscript. 


\section{Acknowledgments}

This study was supported by the French National Research Agency, under reference N 2010 PRSP 00801

\section{Author details}

'EA 4275, Biostatistics, Pharmacoepidemiology and Subjective Measures in Health Sciences, University of Nantes, Nantes, France. ${ }^{2}$ EA 4360 Apemac, Lorraine University, Paris Descartes University, Nancy, France. ${ }^{3}$ INSERM 669 Université Paris-Sud and Université Paris Descartes, Paris, France. ${ }^{4}$ AP-HP, Hôpital Paul Brousse, Département de santé publique, Villejuif, France.

Received: 18 December 2013 Accepted: 27 June 2014

Published: 5 July 2014

\section{References}

1. Smith EM, Pang H, Cirrincione C, Fleishman S, Paskett ED, Ahles T, Bressler LR, Fadul CE, Knox C, Le-Lindqwister N, Gilman PB, Shapiro CL, Alliance for Clinical Trials in Oncology: Effect of duloxetine on pain, function, and quality of life among patients with chemotherapy-induced painful peripheral neuropathy: a randomized clinical trial. JAMA 2013, 309:1359-1367.

2. Lamy A, Devereaux PJ, Prabhakaran D, Taggart DP, Hu S, Paolasso E, Straka Z, Piegas LS, Akar AR, Jain AR, Noiseux N, Padmanabhan C, Bahamondes JC, Novick RJ, Vaijyanath P, Reddy SK, Tao L, Olavegogeascoechea PA, Airan B, Sulling TA, Whitlock RP, Ou Y, Pogue J, Chrolavicius S, Yusuf S, CORONARY Investigators: Effects of off-pump and on-pump coronary-artery bypass grafting at 1 year. N Engl J Med 2013, 368:1179-1188.

3. Cunningham MA, Swanson V, Holdsworth RJ, O'Carroll RE: Late effects of a brief psychological intervention in patients with intermittent claudication in a randomized clinical trial. Br J Surg 2013, 100:756-760.

4. Cartwright M, Hirani SP, Rixon L, Beynon M, Doll H, Bower P, Bardsley M, Steventon A, Knapp M, Henderson C, Rogers A, Sanders C, Fitzpatrick R, Barlow J, Newman SP, Whole Systems Demonstrator Evaluation Team: Effect of telehealth on quality of life and psychological outcomes over 12 months (Whole Systems Demonstrator telehealth questionnaire study): nested study of patient reported outcomes in a pragmatic, cluster randomised controlled trial. BMJ 2013, 346:f653.

5. Weis J, Arraras Jl, Conroy T, Efficace F, Fleissner C, Görög A, Hammerlid E, Holzner B, Jones L, Lanceley A, Singer S, Wirtz M, Flechtner H, Bottomley A: Development of an EORTC quality of life phase III module measuring cancer-related fatigue (EORTC QLQ-FA13). Psychooncology 2013, 22:1002-1007

6. Cella D, Riley W, Stone A, Rothrock N, Reeve B, Yount S, Amtmann D, Bode R, Buysse D, Choi S, Cook K, Devellis R, DeWalt D, Fries JF, Gershon R, Hahn EA, Lai JS, Pilkonis P, Revicki D, Rose M, Weinfurt K, Hays R: The patient-reported outcomes measurement information system (PROMIS) developed and tested its first wave of adult self-reported health outcome item banks: 2005-2008. J Clin Epidemio/ 2010, 63:1179-1194.

7. Langer MM, Hill CD, Thissen D, Burwinkle TM, Varni JW, DeWalt DA: Item response theory detected differential item functioning between healthy and ill children in quality-of-life measures. J Clin Epidemio/ 2008, 61:268-276

8. Fisher GH, Molenaar IW: Rasch Models, Foundations, Recent Developments, and Applications. New-York: Springer-Verlag; 1995.

9. Sébille V, Hardouin J-B, Mesbah M: Sequential analysis of latent variables using mixed-effect latent variable models: impact of non-informative and informative missing data. Stat Med 2007, 26:4889-4904.

10. Hardouin JB, Conroy R, Sébille V: Imputation by the mean score should be avoided when validating a patient reported outcomes questionnaire by Rasch model in presence of informative missing data. BMC Med Res Meth 2011, 11:105.

11. De Bock E, Hardouin JB, Blanchin M, Le Neel T, Kubis G, Bonnaud-Antignac A, Dantan E, Sébille V: Rasch-family models are more valuable than score-based approaches for analyzing longitudinal PRO with missing data. Stat Meth Med Res. in press.

12. Andrich D: Rating scales and rasch measurement. Expert Rev Pharmacoecon Outcomes Res 2011, 11:571-585.

13. Ravens-Sieberer U, Herdman M, Devine J, Otto C, Bullinger M, Rose M, Klasen F: The European KIDSCREEN approach to measure quality of life and well-being in children: development, current application, and future advances. Qual Life Res. in press.
14. Waller J, Ostini R, Marlow LAV, McCaffery K, Zimet G: Validation of a measure of knowledge about human papillomavirus (HPV) using item response theory and classical test theory. Prev Med 2013, 56:35-40

15. Sapin C, Simeoni M-C, El Khammar M, Antoniotti S, Auquier P: Reliability and validity of the VSP-A, a health-related quality of life instrument for ill and healthy adolescents. J Adolesc Health 2005, 36:327-336.

16. Begg C, Cho M, Eastwood S, Horton R, Moher D, Olkin I, Pitkin R, Rennie D, Schulz KF, Simel D, Stroup DF: Improving the quality of reporting of randomized controlled trials: the CONSORT statement. JAMA 1996, 276:637-639.

17. Schulz KF, Altman DG, Moher D, CONSORT Group: CONSORT: Statement: Updated Guidelines for Reporting Parallel Group Randomized Trials. Ann Intern Med 2010, 2010(152):726-732.

18. von Elm E, Altman DG, Egger M, Pocock SJ, Gotzsche PC, Vandenbroucke JP: The Strengthening the Reporting of Observational Studies in Epidemiology (STROBE) statement: guidelines for reporting observational studies. Ann Intern Med 2007, 147:573-577.

19. Des Jarlais DC, Lyles C, Crepaz N: Improving the reporting quality of nonrandomized evaluations of behavioral and public health interventions: the TREND statement. Am J Public Health 2004, 94:361-366.

20. Calvert M, Blazeby J, Altman DG, Revicki DA, Moher D, Brundage MD, CONSORT PRO Group: Reporting of patient-reported outcomes in randomized trials: the CONSORT PRO extension. JAMA 2013, 309:814-822.

21. US Department of Health and Human Services: Guidance for Industry (Patient-reported outcome measures: use in medical product development to support labeling claims). http://www.fda.gov/downloads/Drugs/ GuidanceComplianceRegulatoryInformation/Guidances/UCM193282.pdf.

22. Sébille V, Hardouin J-B, Le Neel T, Kubis G, Boyer F, Guillemin F, Falissard B: Methodological issues regarding power of classical test theory and IRT-based approaches for the comparison of Patient-Reported Outcome measures - A simulation study. BMC Med Res Meth 2010, 10:24.

23. Hardouin J-B, Amri S, Feddag M-L, Sébille V: Towards power and sample size calculations for the comparison of two groups of patients with item response theory models. Stat Med 2012, 31:1277-1290.

24. Julious SA: Sample sizes for clinical trials with normal data. Stat Med 2004 30:1921-1986

25. Glas CAW: The derivation of some tests for the Rasch model from the multinomial distribution. Psychometrika 1988, 53:525-546.

26. Blanchin M, Hardouin J-B, Le Neel T, Kubis G, Blanchard C, Miraillé E, Sébille V: Comparison of CTT and IRT based-approach for the analysis of longitudinal Patient Reported Outcome. Stat Med 2011, 30:825-838.

27. Hardouin J-B, Audureau E, Leplège A, Coste J: Spatio-temporal Rasch analysis of Quality of life outcomes in the french general population. Measurement invariance and group comparisons. BMC Med Res Meth 2012, 212:182

28. Feddag ML, Blanchin M, Hardouin JB, Sébille V: Power analysis on the time effect for the longitudinal Rasch model. J Appl Meas 2014. in press.

doi:10.1186/1471-2288-14-87

Cite this article as: Sébille et al:: A simple ratio-based approach for power and sample size determination for 2-group comparison using Rasch models. BMC Medical Research Methodology 2014 14:87.

\section{Submit your next manuscript to BioMed Central and take full advantage of:}

- Convenient online submission

- Thorough peer review

- No space constraints or color figure charges

- Immediate publication on acceptance

- Inclusion in PubMed, CAS, Scopus and Google Scholar

- Research which is freely available for redistribution 